\title{
Exploiting redox activity of MIL-100(Fe) carrier enables carvacrol prolonged antimicrobial activity
}

Katia Caamaño, ${ }^{a}$ Joaquín Calbo, ${ }^{a}$ Raquel Heras-Mozos, ${ }^{b}$ João C. Waerenborgh, ${ }^{c}$ Bruno J. C Vieira, ${ }^{c}$ Pilar M. Hernández-Muñoz, ${ }^{b}$ Rafael Gavara ${ }^{b}$ and Mónica Giménez-Marqués ${ }^{*}$.

a. Instituto de Ciencia Molecular (ICMol), Universidad de Valencia, c/ Catedrático José Beltrán 2, 46980 Paterna, Spain.

b. Instituto de Agroquímica y Tecnología de Alimentos, IATA-CSIC, Av. Agustín Escardino 7 , 46980 Paterna, Spain.

c. C2TN, DECN, Instituto Superior Técnico, Universidade de Lisboa, EN10, P-2695-066 Bobadela LRS, Portugal.

KEYWORDS: MOFs; controlled delivery; biocomposites; antimicrobial activity; food packaging.

ABSTRACT: The design of efficient food contact materials that maintain optimal levels of food safety is of paramount relevance to reduce the increasing foodborne illnesses. In this work, we develop a smart composite MOF-based material that fosters a unique prolonged antibacterial activity. The composite is obtained by entrapping a natural preserving food molecule, carvacrol, into the mesoporous MIL-100(Fe) material following a direct and biocompatible impregnation method and obtaining particularly high payloads. By exploiting the intrinsic redox nature of MIL-100(Fe) material it is possible to achieve a prolonged activity against E. coli bacteria due to a triggered two-step carvacrol release of films containing the carvacrol@MOF composite. Essentially, it was discovered that based on the underlying chemical interaction among MIL-100(Fe) and carvacrol, it is possible to undergo a reversible charge transfer process between the metallic MOF counterpart and the carvacrol upon certain physical stimuli. During this process, the preferred carvacrol binding site has been monitored by IR, Mössbauer and EPR spectroscopies and is supported by theoretical calculations.

INTRODUCTION. Ensuring food quality and safety is a major global challenge in a society severely affected by foodborne diseases. In this context, naturally occurring bioactive compounds (BAC) (e.g., antioxidants, vitamins, polyphenols) are effectively used as food flavouring agents and/or preservatives to inhibit microbial growth. ${ }^{1}$ Among them, phenolic compounds have recently attracted attention due to their antioxidant and antitumour activity., ${ }^{2,3}$ Despite exhibiting these properties, a desired large-scale use of these compounds is hampered by their volatile and insoluble nature, their susceptibility to various environmental and processing conditions and their characteristic strong aroma. For these reasons, the encapsulation of 
volatile preservatives into carrier materials has been widely investigated in the food industry and biomedical field as a plausible solution to optimize their activity.,5

Nanometric porous Metal-Organic Frameworks (nanoMOFs) have recently emerged as a promising alternative to ensure the safety and quality of food owing to their excellent porosity, high loading capacity, controlled release ability and ease of surface modification.${ }^{6,7}$ In addition, some MOFs are biocompatible, processable and can be produced in large scale, fulfilling industrial requirements while being environmentally responsible. ${ }^{8.9}$ Linked to their performance as encapsulating agents, MOFs have been recently evaluated in the field of food safety for the removal of contaminants from production sources,${ }^{10}$ in food packaging, ${ }^{11}$ improving the preservation of food, ${ }^{12}$ and in the detection and monitoring of contaminants in food products, ${ }^{13}$ among others. As compared to classical carrier agents like nano- and micro-emulsions, lipid nanoparticles or liposomes, MOFs result particularly interesting since not only provide a high drug loading but also gather a controlled release. ${ }^{14,15}$ This is due to the high and regular porosity found in the MOF structures as well as the multiple organic/inorganic groups available for interaction with the guests. As a result, it is possible to tune the uptake and delivery of guest molecules by appropriate selection of the MOF scaffold. This chemical control provides a potential way to design carrier materials for programmed release of multiple active ingredients occurring upon certain physical (light, temperature, humidity) or chemical stimuli ( $\mathrm{pH}$, chelating agents). ${ }^{16}$ Particular examples in the field of food safety include the encapsulation of eugenol in a PUM168 single crystal ${ }^{17}$ or the triggered release of allyl isothiocyanate..$^{18,19}$

Among the possible MOFs to be used as encapsulating agents in food related applications, the mesoporous iron(III) trimesate nanoMOF MIL-100(Fe) offers unique possibilities since is biocompatible and can be produced through green synthesis ${ }^{20}$ in optimal scales..$^{21,22}$ In addition, the structure characteristics of MIL-100(Fe) permit to form accessible coordinatively unsaturated iron sites upon induced reducibility on the framework, strongly modifying the preferred interactions with guest molecules. ${ }^{23}$ Moreover, the nano-structuration of the framework improves its chemical and colloidal stability, thus facilitating the processing of the material for immediate applications like its integration in films. ${ }^{24}$

In this work, a composite material based on a phenolic compound, carvacrol, loaded into nanoMIL$100(\mathrm{Fe})$ is obtained. Carvacrol is an aromatic monoterpene present in the essential oil extracted mostly from oregano which exhibits antimicrobial properties. ${ }^{25-29}$ The MOF scaffold not only stabilizes and protects the active molecule, but also promotes a prolonged release when supported in polymeric films (Figure 1), resulting in a stable antimicrobial effect largely desired in food industry. 


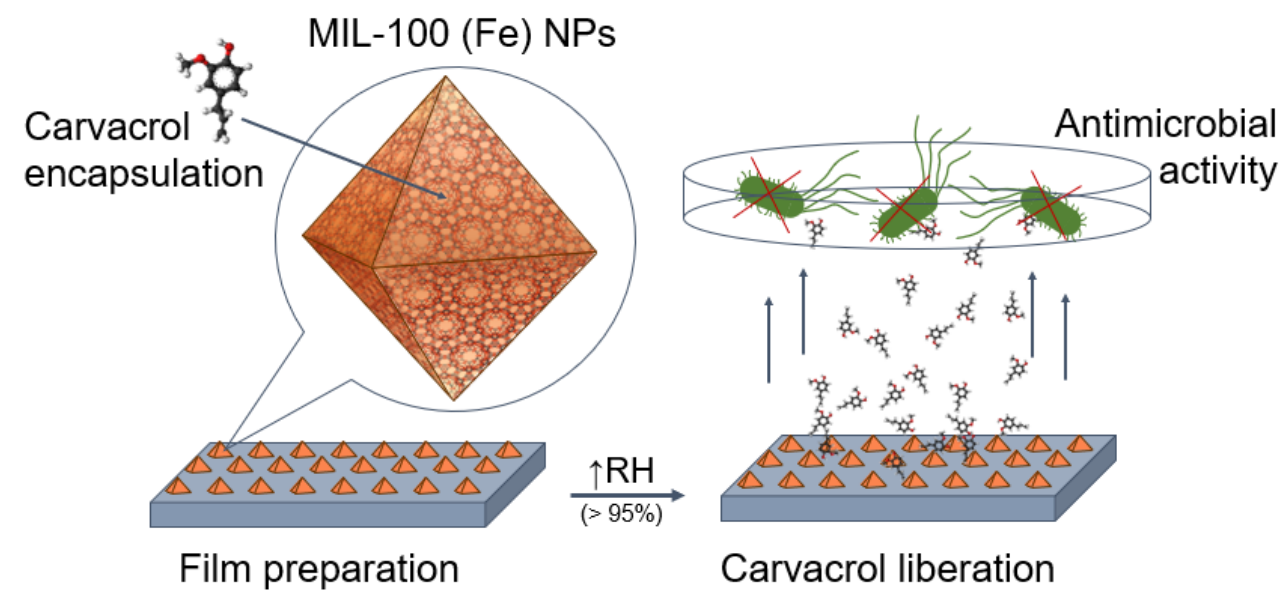

Figure 1: Schematic representation of the encapsulation process and the implementation in polymeric films, showcasing the antimicrobial properties of the liberated carvacrol molecules.

\section{MATERIALS AND METHODS}

Materials. Iron(III) chloride hexahydrate (97\%), 1,3,5-benzene tricaboxylic acid (95\%), and carvacrol (98\%) were purchased from Sigma-Aldrich, Alfa-Aesar, and TCI, respectively. Ethanol absolute was purchased from Honeywell. All chemicals were used as received without need of further purification and Milli-Q water was obtained from a Millipore Milli-Q system.

Synthesis of MIL-100(Fe) nanoparticles. MIL-100(Fe) nanoparticles were synthesized following the procedure described by García Márquez and coworkers ${ }^{20}$ by a microwave-assisted method. The assynthesized material was treated in a KF $0.1 \mathrm{M}$ solution for $2 \mathrm{~h}$ and washed with $\mathrm{H}_{2} \mathrm{O}$ to remove excess of reagents. The particles were collected by centrifugation (9900 rpm, $20 \mathrm{~min})$ and stored.

Preparation of carvacrol@MIL-100(Fe) composite material. Composite carvacrol@MIL-100(Fe) material was obtained following a direct impregnation method. $23 \mathrm{~mL}$ from a $10 \mathrm{mg} \cdot \mathrm{mL}^{-1}$ carvacrol emulsion prepared in a $\mathrm{H}_{2} \mathrm{O}: \mathrm{EtOH}$ (4:1) mixture was directly added to MIL-100(Fe) nanoparticles (weighted humid and corresponding to $200 \mathrm{mg}$ of dried MOF). After 5 days stirring in a $360^{\circ}$ rotating shaker, the carvacrol@MIL-100(Fe) material was retrieved by centrifugation (10000 rpm, 20 min) and dried at air overnight.

Determination of loading capacity (LC) and encapsulation efficiency (EE). Carvacrol content in the obtained composite was determined by thermic desorption Gas Chromatography, using a HP 7890B equipped with a HP5 column of $30 \mathrm{~m}, 320 \mu \mathrm{m}$ of diameter and $0.25 \mu \mathrm{m}$ of thickness. The thermic gradient employed was: $40{ }^{\circ} \mathrm{C}(3 \mathrm{~min}), 10^{\circ} \mathrm{C} \cdot \mathrm{min}^{-1} \mathrm{ramp}$ until $200{ }^{\circ} \mathrm{C}$ and $15 \mathrm{~min}$ isotherm. Injector heats on ballistic 
ramp $\left(600{ }^{\circ} \mathrm{C} \cdot \mathrm{min}^{-1}\right)$ from 40 to $200{ }^{\circ} \mathrm{C}$ and $4 \mathrm{~min}$ isotherm. Equations used for calculations of loading capacity and encapsulation efficiency are further described in the supporting material.

Characterization of carvacrol@MIL-100(Fe) composite material. Dynamic light scattering and $\zeta$ potential measurements were collected in a Zetasizer Ultra (Malvern Panalytical). For transmission electron microscopy a JEM 1010 (JEOL) microscope was employed. X-ray powder diffraction experiments were acquired in an X-ray diffractometer (PANalytical Empyrean) with copper as a radiation source $(\mathrm{Cu}-\mathrm{K} \alpha$, $1.5418 \AA$ ). Infrared spectroscopy spectra were registered employing an ALPHA II FTIR spectrometer (Bruker). Thermogravimetric analyses were carried out with TGA 550 (TA Instruments) in high-resolution mode (Ramp: $20.0{ }^{\circ} \mathrm{C} / \mathrm{min}$ to $680.00{ }^{\circ} \mathrm{C}$; Res 4 Sensitivity 3). $\mathrm{N}_{2}$ adsorption isotherms were obtained using a TRISTAR II apparatus (Micromeritics) at $-196{ }^{\circ} \mathrm{C}$. All samples were activated at $100{ }^{\circ} \mathrm{C}$ under vacuum for $3 \mathrm{~h}$ before measurement. Mössbauer spectra were collected using a conventional constant acceleration spectrometer and a $57 \mathrm{Co}(\mathrm{Rh})$ source. The velocity scale was calibrated with an $\alpha$-Fe foil. Low-temperature measurements were performed with the samples immersed in He exchange gas in a bath cryostat. The spectra were fitted to distributions of quadrupole doublets according to the histogram method. ${ }^{30}$ Electron paramagnetic resonance spectra were collected with a Bruker ELEXYS E580 spectrometer operating in the X-band $(9.47 \mathrm{GHz})$. Sweep width $4960.0 \mathrm{G}$, time constant $2.56 \mathrm{~ms}$, modulation frequency $100 \mathrm{kHz}$, modulation width $1 \mathrm{G}$, microwave power $19.82 \mathrm{~mW}$. EPR spectra were collected with a Bruker ELEXYS E580 spectrometer operating in the X-band $(9.47 \mathrm{GHz})$. Sweep width $4960.0 \mathrm{G}$, time constant $2.56 \mathrm{~ms}$, modulation frequency $100 \mathrm{kHz}$, modulation width $1 \mathrm{G}$, microwave power $19.82 \mathrm{~mW}$.

Preparation of polymeric films. Two different types of zein films were prepared. A $80 \%$ hydroalcoholic solution containing $15 \%$ weight of zein was first prepared. Two fractions were separated: to the first one 0.125 g Carvacrol@MIL-100(Fe) composite was added per g of zein, whereas the corresponding amount of carvacrol was directly added in a concentration of $0.05 \mathrm{~g} / \mathrm{g}$ zein to the second fraction. The mixtures were spread on a flat surface of PTFE using a $200 \mu \mathrm{m}$ coating rod and dried in an open oven at $75^{\circ} \mathrm{C}$ for $10 \mathrm{~min}$. The films obtained were stored in PP/met envelopes until further characterisation. The residual content of carvacrol in both films was analysed. For this, samples were cut from both films, introduced into a micro vial, and tested by thermal desorption and gas chromatography obtaining a final concentration of carvacrol of $0.059 \pm 0.008 \mathrm{~g} / \mathrm{g}$ in the encapsulated film and $0.050 \pm 0.001 \mathrm{~g} / \mathrm{g}$ in the film with pure carvacrol.

Carvacrol release in the films. Relative cumulative release profiles were measured by monitoring the carvacrol release from the polymeric films at $23 \pm 1{ }^{\circ} \mathrm{C}$ and $95 \pm 3 \% \mathrm{RH}$ (simulating the exposure to fresh food). A piece sample of film was placed on a desorption tube where a humid $15 \mathrm{~mL} / \mathrm{min}$ stream of $\mathrm{He}$ hauled the released product to a gas chromatograph. The carvacrol release flow was determined using a HP5890 gas chromatograph with a 200- $\mu \mathrm{L}$ automatic injection valve and a HP5 column of $30 \mathrm{~m}, 320 \mu \mathrm{m}$ 
of diameter and $0.25 \mu \mathrm{m}$ of thickness. Thermal conditions were: $80^{\circ} \mathrm{C}$ at the injection valve, $200{ }^{\circ} \mathrm{C}$ at the injection port, $220^{\circ} \mathrm{C}$ at the flame ionization detector, and $100{ }^{\circ} \mathrm{C}$ at the column oven. Film samples were exposed to high humidity conditions with a He stream that.

Antimicrobial Properties of the Films. Bacterial strain Escherichia coli CECT 434 (ATCC 25922) was obtained from the Spanish Type Culture Collection (Valencia, Spain). This strain was chosen as a Gramnegative model because of its relevance in Food Industry. It was stored in liquid medium Tryptone Soy Broth (TSB) supplied from Scharlab (Barcelona, Spain) with $20 \%$ glycerol at $-80{ }^{\circ} \mathrm{C}$ until needed. The stock cultures were maintained by periodic subculture on agar Tryptone Soy Agar (TSA) from Scharlab (Barcelona, Spain) slants at $4{ }^{\circ} \mathrm{C}$ and transferred monthly. To test antimicrobial effect of active films, the microatmosphere method, in which the volatile active compound released from the film to the headspace of the Petri dish interacts with the microorganisms, was carried out. In this method, $100 \mu \mathrm{L}$ of a bacteria suspension containing approximately $10^{7}$ colony forming units (CFU)/mL were spread over the $15 \mathrm{~mL}$ of TSA surface, and a disk of the antimicrobial films ( $80 \mathrm{~mm}$ in diameter) was adhered to the lid of the Petri dish, without direct contact with the microorganism, sealed with Parafilm and incubated at $37{ }^{\circ} \mathrm{C}$ for $24 \mathrm{~h}$. After the incubation period, the diameter of the resulting inhibition zone in the bacterial growth was measured. ${ }^{31}$ Controls without films and control films with free carvacrol were also tested. Finally, the inoculated Petri dishes were employed to count the colony forming units (CFU) and log reduction value (LRV). For that, agar medium was aseptically removed from the Petri dishes and homogenized in a sterile BagPage with $100 \mathrm{~mL}$ of peptone water for $2 \mathrm{~min}$ with a Stomacher. Serial dilutions were made with peptone water and plated in Petri dishes with $15 \mathrm{~mL}$ of selective agar medium Brilliant Green Agar from Scharlab (Barcelona, Spain). Plates were incubated at $37{ }^{\circ} \mathrm{C}$ for $24 \mathrm{~h}$. Results were expressed as $\log$ CFU/mL. LRV was calculated by comparison between control sample and samples with film (LRV= (Control $\log \mathrm{CFU} / \mathrm{mL})$-(Film $\log \mathrm{CFU} / \mathrm{mL})$. The experiment was carried out in triplicate.

Computational Details. Theoretical calculations were performed under the density functional theory (DFT) framework by means of the Gaussian-16.A03 suite of programs. ${ }^{32}$ Minimum-energy geometry structures were obtained upon atom relaxation at the B3LYP/6-31G(d,p) level of theory ${ }^{33,34}$ including dispersion corrections by means of the Grimme's D3 (Becke-Johnson damping function) protocol. ${ }^{35,36}$ To maintain the topology of the cluster models as in the corresponding MOF, the terminal hydrogen atoms were frozen during the optimization procedure. Non-covalent interaction (NCI) surfaces were obtained under the NCIPLOT-3.0 program $^{37}$ and visualized through the VMD software ${ }^{38}$ with standard thresholds of 0.3 and 0.04 a.u. for the reduced density gradient and density, respectively. Time-dependent DFT calculations were performed on the lowest-lying singlet excited states at the B3LYP/6-31G(d,p) level. Charge analysis was performed through the natural bond order (NBO) approach by means of the NBO 
version 3 as implemented in Gaussian-16.A03. Spin density contours were plotted through the Chemcraft software. ${ }^{39}$

\section{RESULTS AND DISCUSION}

Carvacrol encapsulation in MIL-100(Fe) nanoparticles. To encapsulate the natural preserving food molecule, carvacrol, we followed a simple green method consisting of the direct impregnation of previously obtained MIL-100(Fe) nanoparticles with a concentrated carvacrol solution (Figure 2a). Different experimental parameters including solvents and reaction times were investigated (Table S1). It was found that carvacrol encapsulation was favoured under certain aqueous-alcoholic mixtures in which carvacrol is poorly soluble and forms a milky emulsion, this parameter resulting key for a successful encapsulation. Optimal carvacrol loading was achieved by soaking MIL-100(Fe) nanoparticles into a $10 \mathrm{mg} \cdot \mathrm{mL}^{-1}$ carvacrol ethanolic solution (20\%) with a 5:1 carvacrol:MIL-100(Fe) molar ratio. First evidence of effective carvacrol loading was noticed by a colour change in the powder from orange to dark brown, respectively for bare MIL-100(Fe) and the collected carvacrol@MIL-100(Fe) composite (Figure S1). Encapsulation completion was determined upon monitoring of the carvacrol sorption equilibrium over time in the collected composite material by gas chromatography (Figure $2 \mathrm{~b}$ ). A maximum loading capacity of $42 \%$ was reached after 5 days of immersion, with no further loading occurring up to 10 days (\% calculated as loaded mass of carvacrol per total mass of dry composite), resulting in an encapsulation efficiency of 58\%. This exceptional payload is, to the best of our knowledge, the highest carvacrol uptake onto a MOF obtained in a liquidphase encapsulation, surpassing the considerable 34\% of carvacrol loading achieved in MIL-53(Al) by supercritical $\mathrm{CO}_{2}$ encapsulation. ${ }^{40}$ In addition, as compared to other classical carrier agents, the obtained carvacrol@MIL-100(Fe) composite is in a competitive position in terms of carvacrol loading, only being surpassed by carvacrol-loaded human serum albumin nanoparticles (Table S2). ${ }^{41}$

Carvacrol loading was further evaluated by thermogravimetric analysis (TGA), X-ray powder diffraction (XRPD), $\mathrm{N}_{2}$ adsorption measurements and Fourier transform infrared (FTIR) spectroscopy. Thermal analysis (Figures 2c and S2, Table S2) of the loaded carvacrol@MIL-100(Fe) composite revealed a carvacrol content of $26.7 \mathrm{wt} \%$ (with respect to dehydrated MIL-100(Fe) nanoparticles). After removal of volatiles, in the $120-180{ }^{\circ} \mathrm{C}$ interval and at $230{ }^{\circ} \mathrm{C}$, two separated mass losses of $8.3 \%$ and $18.4 \%$ can be respectively distinguished, which may be attributed to the release of carvacrol molecules differently interacting with the framework (i.e. physisorption vs chemisorption).

MOF integrity was evaluated after carvacrol infiltration by means of XRPD analysis performed before and after the encapsulation process (Figure 2d), confirming that MIL-100(Fe) crystal structure is maintained after carvacrol loading. Then, to identify the porous nature of the composite material and therefore determine the external or internal association of the carvacrol molecules, $\mathrm{N}_{2}$ sorption studies were 
conducted before and after carvacrol impregnation (Figure 2e). A significant decrease (ca. 87\%) in the porous surface was evidenced after carvacrol encapsulation $\left(\mathrm{S}_{\mathrm{BET}}=1491 \mathrm{vs} .196 \mathrm{~m}^{2} \cdot \mathrm{g}^{-1}\right.$, respectively for MIL-100(Fe) and carvacrol@MIL-100(Fe)), which is consistent with an effective carvacrol loading.

(a)

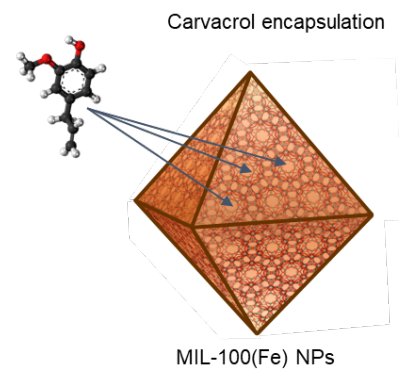

(d)

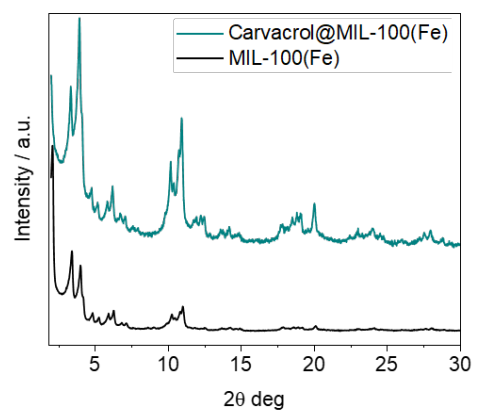

(b)

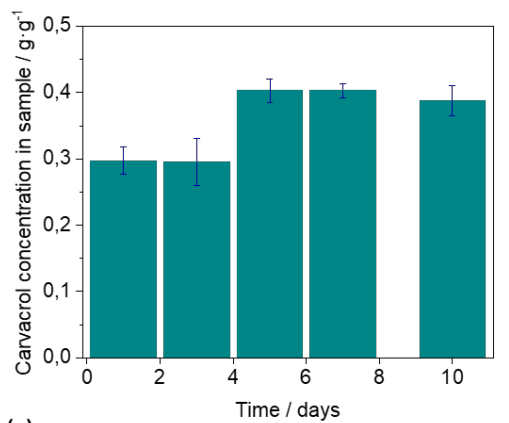

(e)

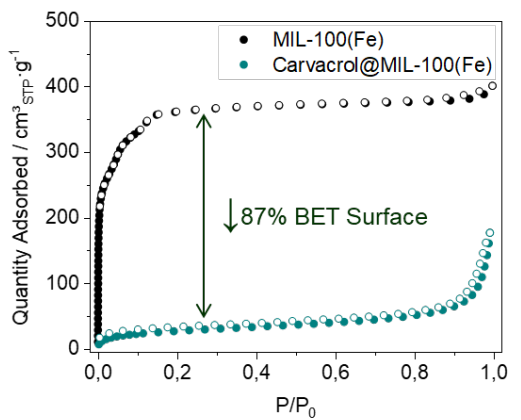

(c)

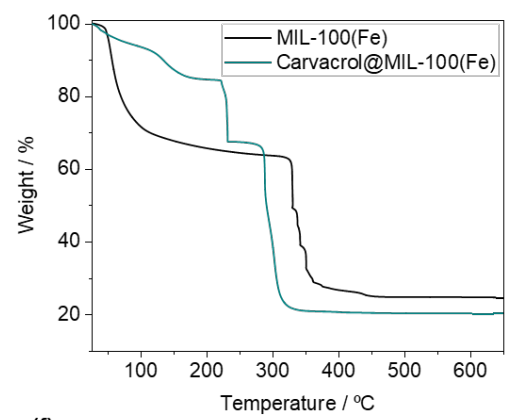

(f)

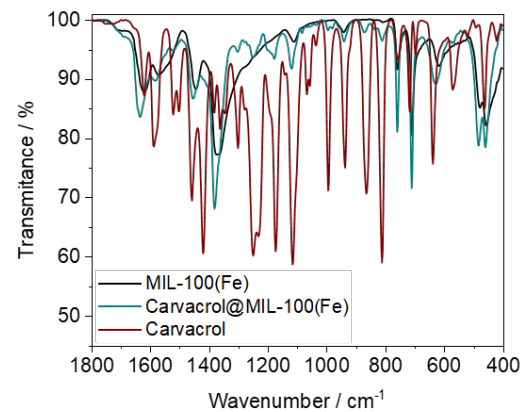

Figure 2. (a) Scheme of carvacrol encapsulation into MIL-100(Fe). (b) Carvacrol content in the composite as a function of time of incubation. (c-f) Characterization of the carvacrol@MIL-100(Fe) composite (green) as compared with MIL-100(Fe) (black): (c) Thermal decomposition profiles. (d) X-ray powder diffractograms. (e) $\mathrm{N}_{2}$ sorption studies at $77 \mathrm{~K}$ (solid symbols for adsorption and open ones for desorption). (f) Selected infrared spectra region (1800-400 $\mathrm{cm}^{-1}$ ) of the composite as compared with MIL-100(Fe) and free carvacrol (red).

Analysis of the FTIR spectra of the impregnated carvacrol@MIL-100(Fe) material (Figures 2f and S3) revealed the appearance of the most remarkable bands of carvacrol $\left(v_{\mathrm{O}-\mathrm{H}} 3361 \mathrm{~cm}^{-1}\right.$ and $v_{\mathrm{C}-\mathrm{H}} 2958$ and 2869 $\mathrm{cm}^{-1}$ ) in addition to the typical bands corresponding to $\mathrm{Fe}^{\mathrm{III}}$ trimesate MOF ( $v_{\mathrm{C}=\mathrm{O}} 1620,1570,1445$, and $\left.1370 \mathrm{~cm}^{-1}\right)$. Moreover, it can be noticed that the $v_{\text {as }}\left(\mathrm{Fe}_{3} \mathrm{O}\right)$ vibration at $618 \mathrm{~cm}^{-1}$, characteristic of the $\mathrm{Fe}^{\mathrm{III}}$ oxo-trimeric core in MIL-100(Fe), ${ }^{42}$ appears shifted to $628 \mathrm{~cm}^{-1}$ in the composite material. This suggests an effective interaction between the carvacrol molecules and the accessible $\mathrm{Fe}^{\mathrm{III}}$ sites, as previously described for the binding of different molecules. ${ }^{43,44}$ In an attempt to further investigate the host-guest interaction, the biocomposite and the control empty material were exposed to thermal treatment under vacuum for 2 hours. Thermal activation $\left(250{ }^{\circ} \mathrm{C}\right)$ has been previously shown to induce the generation of coordinatively unsaturated iron sites (CUS) with mixed valence $\mathrm{Fe}^{\mathrm{II}} / \mathrm{Fe}^{\mathrm{III}}$ trimers in MIL-100(Fe), manifested by a shifting 
of the $v_{\text {as }}\left(\mathrm{Fe}_{3} \mathrm{O}\right)$ band located at $618 \mathrm{~cm}^{-1}$ to $597 \mathrm{~cm}^{-1}$ after thermal treatment in IR spectroscopy studies. ${ }^{42,45}$ In a similar study, we limited this high temperature activation to $190{ }^{\circ} \mathrm{C}$ in order to remain below the carvacrol boiling point. Exposing MIL-100(Fe) control to this thermal activation leads to a reduced shifting in the $v_{\text {as }}\left(\mathrm{Fe}_{3} \mathrm{O}\right)$ from 618 to $611 \mathrm{~cm}^{-1}$ recovering the original value upon $1 \mathrm{~min}$ of air exposure (Figure $\mathrm{S} 4$ ). This band evolution reflects a reversible partial loss of coordinated water molecules as compared to the reported material, as may be expected for lower activation temperature and lack of sample isolation from ambient conditions. ${ }^{42,45}$ In case of carvacrol@MIL-100(Fe) treated at $190{ }^{\circ} \mathrm{C}$ under vacuum, a shifting in the $v_{\text {as }}\left(\mathrm{Fe}_{3} \mathrm{O}\right)$ band from 628 to $605 \mathrm{~cm}^{-1}$ is observed, and the original value is recovered after $1 \mathrm{~min}$ of air exposure (Figure 3). The large shifting of the $v_{\text {as }}\left(\mathrm{Fe}_{3} \mathrm{O}\right)$ band observed in the case of the activated composite is in good agreement with the displacement reported in the mixed valence $\mathrm{Fe}^{\mathrm{II}} / \mathrm{Fe}^{\mathrm{III}} \mathrm{MIL}-100(\mathrm{Fe})$ spectrum ( $23 \mathrm{vs} 21 \mathrm{~cm}^{-1}$, respectively). This phenomenon is accompanied by a drastic colour change from brown to an intense black carbon-like colour, this colour change being reversible to some extent upon exposure to air. It is worth mentioning that crystallinity was preserved during this process, as denoted by maintenance of the XRPD patterns in the material (Figure S5).

(a)

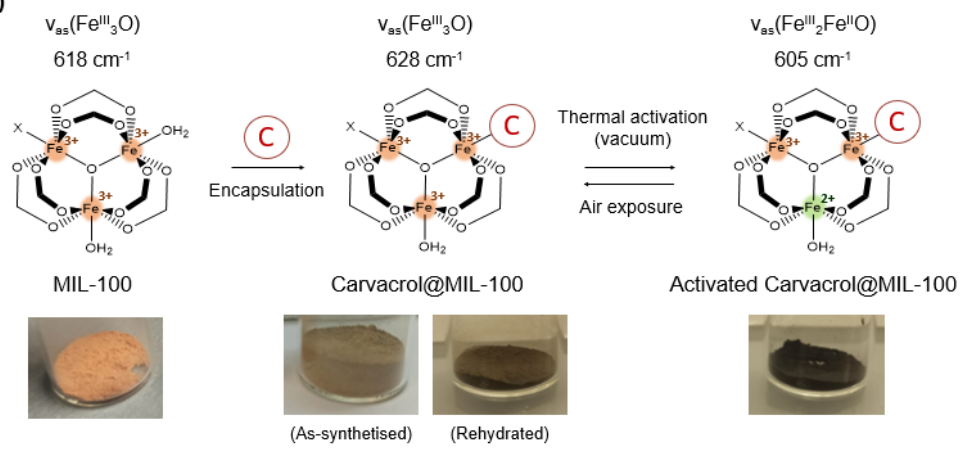

(b)

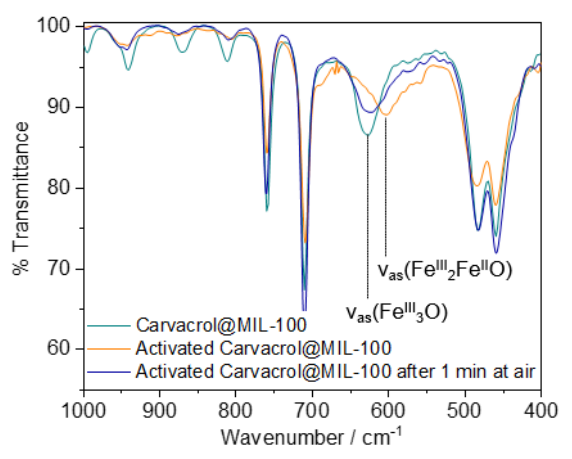

Figure 3. (a) Scheme of (i) encapsulation, (ii) thermal activation under vaccum and (iii) reversible air exposure processes, with the corresponding colour change in each step. Posotion of the IR asymmetric stretching bands of the iron trimeric unit is highlighted. (b) Selected infrared spectra comparison of the composite before activation (green), after thermal treatment under vacuum $\left(190{ }^{\circ} \mathrm{C}\right.$ for 2 hours) (orange) and upon air exposure for 1 minute (blue).

Mössbauer and EPR spectroscopies. In order to evaluate the effect of carvacrol loading in the MIL100(Fe) electronic structure, Mössbauer spectroscopy experiments were carried out. All spectra were collected at $80 \mathrm{~K}$. Control experiments in the as synthesized MIL-100(Fe) and carvacrol@MIL-100(Fe) loaded materials revealed spectra with two broad peaks fitted to distributions of quadrupole doublets (Figure 4). Table S3 summarises the calculated isomer shift (IS) values as well as the temperature independent quadrupole splitting (QS), which are consistent with high-spin $\mathrm{Fe}^{\mathrm{III}}(\mathrm{S}=5 / 2)$ in both 
samples.,20,46-48 In case of the carvacrol@MIL-100(Fe) composite, a lower QS of Fe ${ }^{\mathrm{III}}$ can be detected, which suggests a different $\mathrm{Fe}^{\mathrm{III}}$ environment in the composite. This difference may arise from the replacement of one of the water molecules coordinated to the $\mathrm{Fe}^{\mathrm{III}}$ centres by one molecule of carvacrol, in agreement with the shifting of the $v_{\text {as }}\left(\mathrm{Fe}_{3} \mathrm{O}\right)$ band observed in IR measurements. Studies on the activated compounds were then performed, after exposing MIL-100(Fe) and carvacrol@MIL-100(Fe) samples to thermal treatment $\left(190{ }^{\circ} \mathrm{C}\right)$ under vacuum for 6 hours and quenching the samples in liquid nitrogen for analysis. As expected, activated MIL-100(Fe) spectrum obtained at $80 \mathrm{~K}$ (Figure 4c) showed significantly higher average QS as compared to the pristine sample. This is consistent with a more distorted $\mathrm{Fe}^{\mathrm{III}}$ environment becoming 5coordinated due to the loss of coordinated water molecules.$^{45}$ Once the sample was brought back to room temperature in air, the spectrum of the untreated sample was recovered, confirming the reversibility of the desolvation process. Similar analysis performed on activated carvacrol@MIL-100(Fe) spectrum (Figure $4 \mathrm{~d}$ ) indicates that approximately $24 \%$ of the Fe is reduced to high-spin $\mathrm{Fe}^{\mathrm{II}}$, with the estimated IS and QS suggesting that both $\mathrm{Fe}^{\mathrm{II}}$ and $\mathrm{Fe}^{\mathrm{III}}$ are 6-coordinated. ${ }^{46}$ Then, the initial carvacrol@MIL-100(Fe) spectrum is recovered after exposing the activated sample in air and at room temperature for a few hours. These observations are in agreement with IR experiments and denote the effective reversible formation of the mixed valence trimer in the composite material with retention of the carvacrol molecules coordinated to the $\mathrm{Fe}^{\mathrm{III}}$ centers.
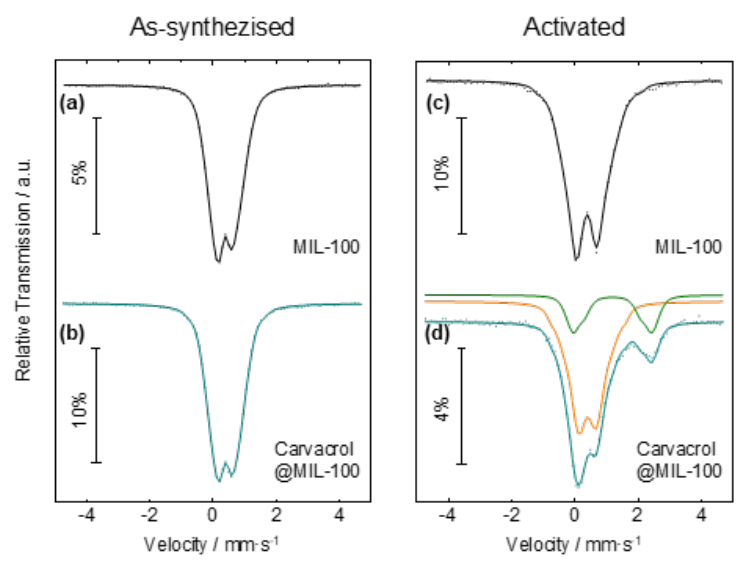

Figure 4: Mössbauer spectra recorded at $80 \mathrm{~K}$ of MIL-100(Fe) (black) and carvacrol@MIL-100(Fe) (green) measured as-synthesized (left) and after activation (right). The solid lines over the experimental points are the fitted distribution of quadrupole doublets. The sum of two distributions of quadrupole doublets due to $\mathrm{Fe}^{\mathrm{III}}$ and $\mathrm{Fe}^{\mathrm{II}}$, is shown slightly shifted for clarity in orange and green, respectively.

To further investigate the coordination between carvacrol and MIL-100(Fe), EPR experiments were conducted. Spectra recorded at room temperature revealed similar signals for the as-synthesized materials before and after encapsulation (Figure S6), whereas the appearance of a characteristic radical signal $(\mathrm{g}=$ 
2.005) was observed upon activation of the composite $\left(190^{\circ} \mathrm{C}\right.$ under vacuum for $2 \mathrm{~h}$ ), suggesting a radicalmediated mechanism for the carvacrol coordination to the $\mathrm{Fe}^{\mathrm{III}}$ centres. Interestingly, this radical remains stable with time, as long as the material is isolated from air.

Theoretical calculations. Density functional theory calculations were performed to shed light into the coordination process between carvacrol and the $\mathrm{Fe}^{\mathrm{III}}$ oxo-trimeric core, and the formation of the mixedvalence $\mathrm{Fe}^{\mathrm{II}} / \mathrm{Fe}^{\mathrm{III}} \mathrm{MIL}-100(\mathrm{Fe})$ upon thermal activation. An oxo-centred tri-metallic cluster model with six benzoate ligands was extracted from the crystal structure of MIL-100(Fe), bearing two water molecules and a fluoride anion in the coordination environment of $\mathrm{Fe}^{\mathrm{III}}$ atoms for charge neutrality, and was optimized at the B3LYP/6-31G(d,p) level of theory in a high-spin configuration (Figure S7). A preliminary evaluation of the thermodynamics of $\mathrm{Fe}^{\mathrm{III}}$ coordination bonds, indicates that insertion of carvacrol in MIL-100(Fe) is expected to occur by replacing a coordinative water molecule (Figure S8), this process resulting energetically favoured $(\Delta E=-16.15 \mathrm{kcal} / \mathrm{mol}$ ) (Figure S9). The minimum-energy structure of the oxocentred trimetallic cluster indicates that the hydroxy group of carvacrol effectively coordinates with an $\mathrm{Fe}^{\mathrm{III}}$ atom, displaying a small interatomic distance of $2.29 \AA$ (Figure 5a), similar to that calculated for a coordinating water molecule (2.25 ̊). Non-covalent index (NCI) surfaces evidence a large number of weak but stabilizing dispersion interactions between the carvacrol moiety and the benzoate units of the cluster (green surfaces in Figure 5b), whereas the strong coordination bond between the hydroxy group of carvacrol and one $\mathrm{Fe}^{\mathrm{III}}$ is revealed as a localized bluish NCI surface (Figure 5b inset and Figure S10).

a)

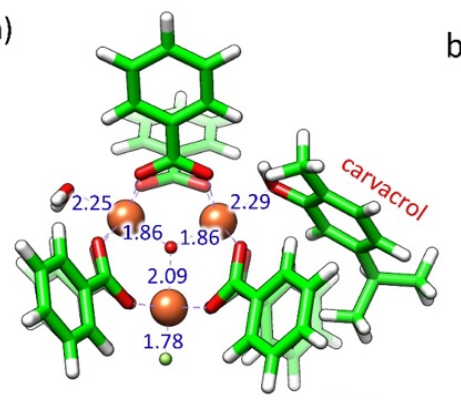

b)

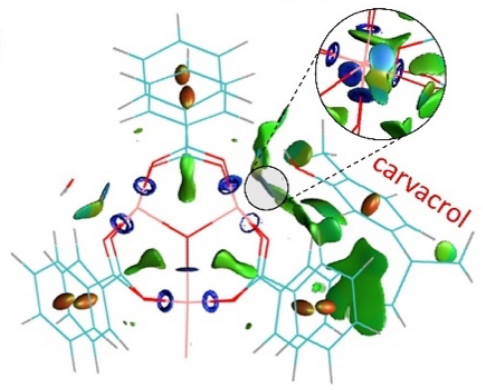

c)

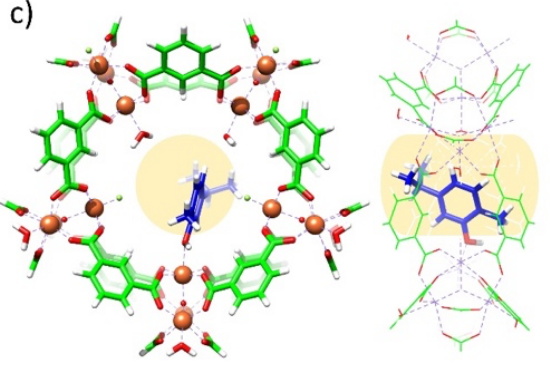

Figure 5. (a) Minimum-energy structure for the representative cluster of MIL-100(Fe) upon carvacrol inclusion. Relevant coordination distances are indicated in $\AA$. Colour coding: $\mathrm{C}$ in green, $\mathrm{O}$ in red, $\mathrm{H}$ in white and Fe in orange. (b) Non-covalent index (NCI) surface showing the large amount of dispersion interactions that stabilize the chemisorption of carvacrol into the MIL-100(Fe) cluster. (c) Front (left) and side (right) views of the minimum-energy structure calculated for the MIL-100(Fe) pentagonal window upon carvacrol complexation. Carvacrol carbon atoms are coloured in blue for better viewing. The window gate is calculated with a diameter of $5.5 \AA$ and coloured in light yellow. 
A bigger cluster consisting of the pentagonal gate of MIL-100(Fe) was modelled to support the structural and stability properties predicted for the coordination of carvacrol. After geometry optimization, a carvacrol molecule was placed inside the pentagonal window to replace a coordinating water molecule (Figure 6c). Theoretical calculations at the B3LYP/6-31G(d,p) level indicate that the coordination of carvacrol is significantly more stable than with water $(\Delta E=-11.23 \mathrm{kcal} / \mathrm{mol}$ for the reaction of replacing a coordinating water by carvacrol), in good accord with that predicted for the smaller cluster model and in line with the experimental evidences. NCI surfaces confirm the presence of a large number of noncovalent interactions between the carvacrol molecule and the framework, along with a strong, localized interaction corresponding to the coordination bond with $\mathrm{Fe}^{\mathrm{III}}$ (see Figure S11). Note that the pentagonal pore gate is predicted with a diameter of $5.5 \AA$, which nicely fits the carvacrol size of $5.0 \AA$ calculated without considering the hydroxy group (see light yellow region in Figure 5c). The hexagonal pore gate, which is of about $8.6 \AA$, is therefore expected to bear and diffuse the carvacrol along the framework easily.

Spin density contours indicate that coordinated carvacrol, in its neutral protonated form, does not show a radical character (oxidized state) that could explain the reduction of one $\mathrm{Fe}^{\mathrm{III}}$ to engender a mixed-valence $\mathrm{Fe}^{\mathrm{II}} / \mathrm{Fe}^{\mathrm{III}}$ system (Figure 6a). Accumulated charges confirm the closed-shell nature of carvacrol moiety, which is barely charged $(+0.08 \mathrm{e}$; Figure S12), similar to that calculated for the coordinated water molecule $(+0.09 \mathrm{e})$. On the other hand, time-dependent DFT calculations predict low-lying singlet electronic transitions in the range of 2.2-2.6 eV from the carvacrol system to the Fe- $d$ orbitals (Figure S13), supporting its electron-donor character compared to the tri-metallic cluster of MIL-100(Fe). Spontaneous one-electron carvacrol oxidation and MIL-100(Fe) reduction was hypothesized to origin upon deprotonation of the relatively acid hydroxy group of carvacrol when coordinated. Theoretical calculations show that proton transfer to other entities is energetically feasible (for example, proton transfer to a coordinating fluoride and water exchange is calculated $(<10 \mathrm{kcal} / \mathrm{mol}$; Figure S14). Upon carvacrol deprotonation, the excess of charge in carvacrol moiety is partially withdrawn by the oxo-centred cluster, especially in one of the iron atoms, thus supporting the formation of a mixed-valence $\mathrm{Fe}^{\mathrm{II}} / \mathrm{Fe}^{\mathrm{III}}$ tri-metallic system (Figure S12). This leads to the formation of a carvacrol radical, which is confirmed by the spin density contours (Figure 6b). The radical carvacrol species coordinated to MIL-100(Fe) is predicted to exhibit several low-lying singlet excited states of charge-transfer nature, with a moderate intensity, and extending over the full visible region of the absorption spectrum (Figure S15). These results are in agreement with the black carbon-like colour displayed by carvacrol@MIL-100(Fe) upon thermal activation. 

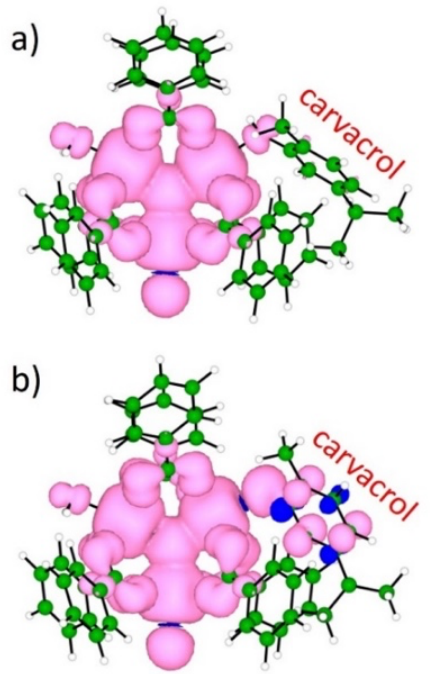

Figure 6. Spin density contours (isovalue $=0.001 \mathrm{au}$ ) calculated for the representative tri-metallic MIL$100(\mathrm{Fe})$ cluster with coordination of a) carvacrol molecule or b) deprotonated carvacrol (radical) system.

Release kinetics of encapsulated carvacrol from films. Carvacrol liberation was studied after the incorporation of the composite in a Zein polymeric matrix simulating fresh food environmental conditions (see Materials and Methods section). Figure 7a shows the results of carvacrol liberation from the compositecontaining film as compared to a control film containing free carvacrol in the same concentration. Control free carvacrol release profile is characterized by a direct continuous delivery with a maximum at $20 \mathrm{~h}$ followed by an exponential decay, which corresponds with a delivery governed by diffusion until agent depletion. A unique profile is observed in the composite-containing film, which is described with a significant carvacrol retention of $c a .5$ hours occurring before the liberation starts. After $5 \mathrm{~h}$, two clearly different delivery phases are observed that are ascribed to the occurrence of sequential fast and slow desorption processes reflected in a first maximum at $20 \mathrm{~h}$, followed by an additional second maximum at $66 \mathrm{~h}$. This behaviour can be explained by attributing each maximum to different host-guest interactions between the carvacrol molecules and the MIL-100(Fe) framework. The first release may correspond to weakly interacting carvacrol molecules, whereas up to two days of high relative humidity conditions are required in order to trigger the liberation of the chemisorbed molecules. This MOF-mediated two-step release represents a clear improvement as compared to the same amount of free molecule in the polymeric film, evidencing the unique MOF capability to deliver carvacrol at prolonged times. 
(a)

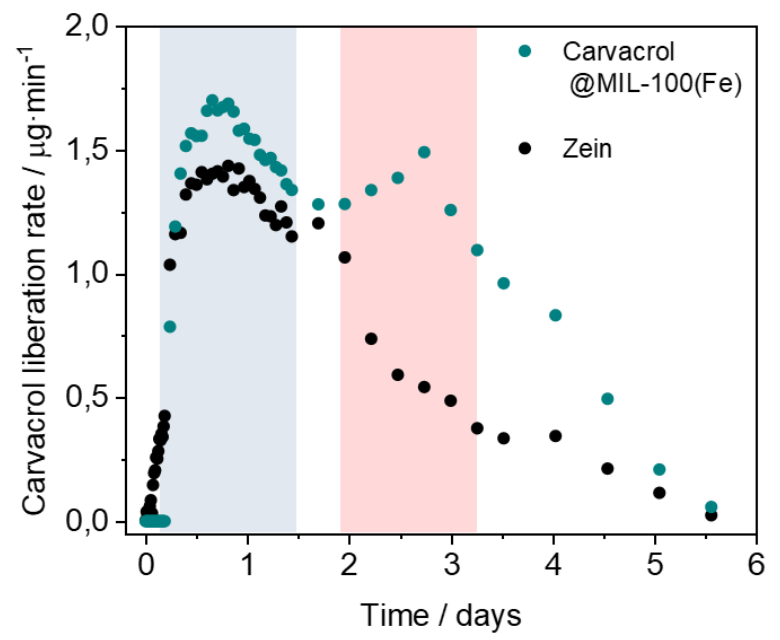

(b)
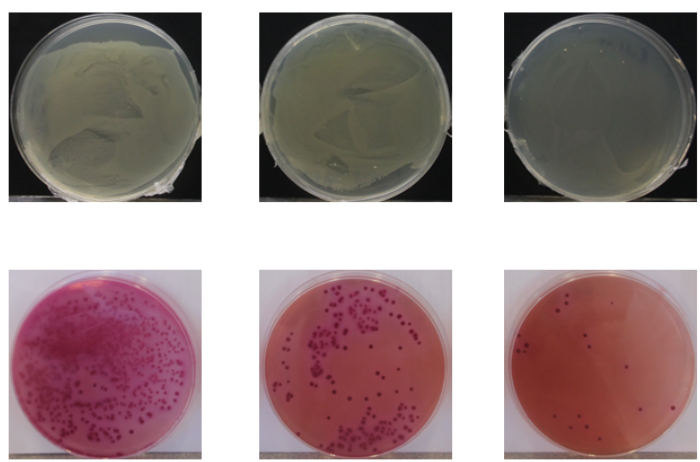

CONTROL

CARVACROL CARVACROL@MIL-100(Fe)

Figure 7. (a) Carvacrol liberation rate in $1 \mathrm{~g}$ of zein film containing carvacrol@MIL-100(Fe) composite. (b) Images of the microatmosphere test carried out to determine the activity of films. On the top, images of the Petri dishes showing the grass density generated by bacteria after the incubation for control (left), carvacrol film (middle) and carvacrol@MIL-100(Fe) containing film (right). On the bottom, images of the Petri dishes with Brilliant green agar after the $5^{\text {th }}$ decimal dilution.

Microbiology studies were conducted in order to determine the efficiency of the obtained composite against bacteria. Three dishes were seeded with E. coli. The first one contained a film of the carvacrol@MIL$100(\mathrm{Fe})$ composite, the second contained a zein film with the same concentration of free carvacrol, and the third one was a control. Figure $7 \mathrm{~b}$ reveals a clear reduction of the grass density occurring on the samples with carvacrol, especially, with the film incorporated with carvacrol@MIL-100(Fe) composite corroborating the antimicrobial effect of the active films. Quantitative effects of the active films (see Table 1) expose a clear reduction on the bacterial growth with respect control, with a $90 \%$ decrease in the number of colony-forming units (CFU) in the case of free carvacrol film, and $98.3 \%$ of $\mathrm{CFU}$ reduction for the carvacrol@MOF composite film. This translates into a $60 \%$ increase in the antimicrobial effect of carvacrol when encapsulated into the MIL-100(Fe) framework, compared to the addition of the same amount of free carvacrol in the film.

Table 1. Antimicrobial activity of films ( $80 \mathrm{~mm}$ diameter surface) against E. coli. Data are colony forming units observed after incubation in Preti dishes without film (control), free carvacrol film and carvacrol@MOF composite.

\section{Sample \\ E.coli}

\section{$(\log \mathrm{CFU} / \mathrm{mL})$}

\section{Log reduction value}




\begin{tabular}{lll}
\hline Control & $9.04 \pm 0.12$ & \\
\hline Free carvacrol film & $8.02 \pm 0.21$ & 1.02 \\
\hline $\begin{array}{l}\text { Carvacrol@MOF } \\
\text { composite film }\end{array}$ & $7.28 \pm 0.11$ & 1.76 \\
\hline
\end{tabular}

\section{SUMMARY AND CONCLUSIONS}

A carvacrol@MIL-100(Fe) composite containing considerable payloads of active agent was prepared following a direct impregnation method compatible with food related uses. In addition to provide chemical stability to the active molecule, MIL-100(Fe) scaffold endorses a remarkable sustained delivery when processed in polymeric films, as a result of its unique redox responsiveness that promote effective interactions with the host molecule. Mossbauer spectroscopy supported by theoretical calculations revealed a successful reversible interaction of the carvacrol molecules with the redox-active MIL-100(Fe) scaffold, thus enabling a prolonged delivery. The released carvacrol dose was enough to fight bacterial pathogens, with an improved activity against $E$. Coli in comparison with an equivalent "free" carvacrol dosage. The combination of a direct preparation, the facile processing and the scaffold-mediated delivery performance that enables extended carvacrol bactericide activity make the obtained carvacrol@MIL-100(Fe) composite a promising candidate for food packaging applications.

\section{ASSOCIATED CONTENT}

Supporting Information. Additional experimental details, materials, and methods, including photographs of experimental setup. This material is available free of charge via the Internet at http://pubs.acs.org

\section{AUTHOR INFORMATION}

\section{Corresponding Author}

e-mail: monica.gimenez-marques@uv.es

\section{Author Contributions}

K.C. performed the experimental work (unless specified otherwise) and wrote the original draft. J.C.W. and B.J.C.V. performed the Mössbauer spectroscopy experiments and analysed the results. Theoretical calculations and discussions were carried out by J.C. Gas Chromatography measurements, film liberation profiles and microbiology studies were conducted by R.G., P.M.H. and R.H.M. All authors discussed the results and commented on the manuscript. M.G.M. conceived the project and supervised the work. 


\title{
Notes
}

The authors declare no competing financial interest

\section{ACKNOWLEDGMENTS}

The authors acknowledge funding from MCIN/AEI/10.13039/501100011033 (grant PID2020-119748GAI00 and CEX2019-000919-M, grant RTI2018-093452-B-I00 and PRE2018-083355 included in Project MAT2017-89993-R funded by "ERDF A way of making Europe”, and grant BES-2016-077380 funded by "ESF Investing in your future") and from Generalitat Valenciana (SEJI2020/036 and GV/2021/027). IST authors acknowledge FCT (Portugal) support through contracts UID/Multi/04349/2019 and PTDC/QUIQIN/32240/2017.

\begin{abstract}
ABBREVIATIONS
BAC, bioactive compounds; MOF, metal-organic framework; CUS, coordinatively unsaturated sites; EO, essential oil; EE, encapsulation efficiency; LC, loading capacity; GC, gas chromatography; DLS, dynamic light scattering; TEM, transmission electron microscopy; XRPD, X-ray powder diffraction; FTIR, Fouriertransform infrared; TGA, thermogravimetric analysis; EPR, electron paramagnetic resonance.
\end{abstract}

\section{REFERENCES}

(1) Amiri, S.; Moghanjougi, Z. M.; Rezazadeh Bari, M.; Khaneghah, A. M. CODON Natural Protective Agents and Their Applications as Bio-Preservatives in the Food Industry: An Overview of Current and Future Applications. Ital. J. Food Sci. 2021, 33 (SP1), 55-68.

(2) Katsube, N.; Iwashita, K.; Tsushida, T.; Yamaki, K.; Kobori, M. Induction of Apoptosis in Cancer Cells by Bilberry (Vaccinium Myrtillus) and the Anthocyanins. J. Agric. Food Chem. 2003, 51 (1), 68-75.

(3) Mukherjee, A.; Basu, S.; Sarkar, N.; Ghosh, A. Advances in Cancer Therapy with Plant Based Natural Products. Curr. Med. Chem. 2012, 8 (12), 1467-1486.

(4) Asbahani, A. El; Miladi, K.; Badri, W.; Sala, M.; Addi, E. H. A.; Casabianca, H.; Mousadik, A. El; Hartmann, D.; Jilale, A.; Renaud, F. N. R.; et al. Essential Oils: From Extraction to Encapsulation. Int. J. Pharm. 2015, 483 (1-2), 220-243. 
(5) Pateiro, M.; Gómez, B.; Munekata, P. E. S.; Barba, F. J.; Putnik, P.; Kovačević, D. B.; Lorenzo, J. M. Nanoencapsulation of Promising Bioactive Compounds to Improve Their Absorption, Stability, Functionality and the Appearance of the Final Food Products. Molecules 2021, 26 (6).

(6) Wang, P.-L.; Xie, L.-H.; Joseph, E. A.; Li, J.-R.; Su, X.-O.; Zhou, H.-C. Metal-Organic Frameworks for Food Safety. Chem. Rev. 2019, 119 (18), 10638-10690.

(7) Shen, M.; Forghani, F.; Kong, X.; Liu, D.; Ye, X.; Chen, S.; Ding, T. Antibacterial Applications of Metal-Organic Frameworks and Their Composites. Compr. Rev. Food Sci. Food Saf. 2020, 19 (4), 13971419.

(8) Bayliss, P. A.; Ibarra, I. A.; Pérez, E.; Yang, S.; Tang, C. C.; Poliakoff, M.; Schröder, M. Synthesis of Metal-Organic Frameworks by Continuous Flow. Green Chem. 2014, 16 (8), 3796-3802.

(9) Avci-Camur, C.; Perez-Carvajal, J.; Imaz, I.; Maspoch, D. Metal Acetylacetonates as a Source of Metals for Aqueous Synthesis of Metal-Organic Frameworks. ACS Sustain. Chem. Eng. 2018, 6 (11), 14554-14560.

(10) Gao, Q.; Xu, J.; Bu, X. H. Recent Advances about Metal-Organic Frameworks in the Removal of Pollutants from Wastewater. Coord. Chem. Rev. 2019, 378, 17-31.

(11) Chopra, S.; Dhumal, S.; Abeli, P.; Beaudry, R.; Almenar, E. Metal-Organic Frameworks Have Utility in Adsorption and Release of Ethylene and 1-Methylcyclopropene in Fresh Produce Packaging. Postharvest Biol. Technol. 2017, 130 (January), 48-55.

(12) Wyszogrodzka, G.; Marszałek, B.; Gil, B.; Dorozyński, P. Metal-Organic Frameworks: Mechanisms of Antibacterial Action and Potential Applications. Drug Discov. Today 2016, 21 (6), 10091018.

(13) Yao, C. X.; Zhao, N.; Liu, J. C.; Chen, L. J.; Liu, J. M.; Fang, G. Z.; Wang, S. Recent Progress on Luminescent Metal-Organic Framework-Involved Hybrid Materials for Rapid Determination of Contaminants in Environment and Food. Polymers (Basel). 2020, 12 (3), 1-29.

(14) Horcajada, P.; Serre, C.; Vallet-Regí, M.; Sebban, M.; Taulelle, F.; Férey, G. Metal-Organic Frameworks as Efficient Materials for Drug Delivery. Angew. Chemie - Int. Ed. 2006, 45 (36), 5974-5978.

(15) Cunha, D.; Ben Yahia, M.; Hall, S.; Miller, S. R.; Chevreau, H.; Elkaïm, E.; Maurin, G.; Horcajada, P.; Serre, C. Rationale of Drug Encapsulation and Release from Biocompatible Porous Metal-Organic Frameworks. Chem. Mater. 2013, 25 (14), 2767-2776. 
(16) Katsoulidis, A. P.; Antypov, D.; Whitehead, G. F. S.; Carrington, E. J.; Adams, D. J.; Berry, N. G.; Darling, G. R.; Dyer, M. S.; Rosseinsky, M. J. Chemical Control of Structure and Guest Uptake by a Conformationally Mobile Porous Material. Nature 2019, 565 (7738), 213-217.

(17) Balestri, D.; Mazzeo, P. P.; Perrone, R.; Fornari, F.; Bianchi, F.; Careri, M.; Bacchi, A.; Pelagatti, P. Deciphering the Supramolecular Organization of Multiple Guests Inside a Microporous MOF to Understand Their Release Profile. Angew. Chemie - Int. Ed. 2021, 10194-10202.

(18) Wang, H.; Lashkari, E.; Lim, H.; Zheng, C.; Emge, T. J.; Gong, Q.; Yam, K.; Li, J. The MoistureTriggered Controlled Release of a Natural Food Preservative from a Microporous Metal-Organic Framework. Chem. Commun. 2016, 52 (10), 2129-2132.

(19) Lashkari, E.; Wang, H.; Liu, L.; Li, J.; Yam, K. Innovative Application of Metal-Organic Frameworks for Encapsulation and Controlled Release of Allyl Isothiocyanate. Food Chem. 2017, 221, 926-935.

(20) García Márquez, A.; Demessence, A.; Platero-Prats, A. E.; Heurtaux, D.; Horcajada, P.; Serre, C.; Chang, J. S.; Férey, G.; De La Peña-O'Shea, V. A.; Boissière, C.; et al. Green Microwave Synthesis of MIL-100(Al, Cr, Fe) Nanoparticles for Thin-Film Elaboration. Eur. J. Inorg. Chem. 2012, 100 (32), 51655174.

(21) Horcajada, P.; Surblé, S.; Serre, C.; Hong, D.-Y.; Seo, Y.-K.; Chang, J.-S.; Grenèche, J.-M.; Margiolaki, I.; Férey, G. Synthesis and Catalytic Properties of MIL-100(Fe), an Iron( <scp>iii </Scp> ) Carboxylate with Large Pores. Chem. Commun. 2007, 100 (27), 2820-2822.

(22) Grall, R.; Hidalgo, T.; Delic, J.; Garcia-Marquez, A.; Chevillard, S.; Horcajada, P. In Vitro Biocompatibility of Mesoporous Metal (III; Fe, Al, Cr) Trimesate MOF Nanocarriers. J. Mater. Chem. B 2015, 3 (42), 8279-8292.

(23) Yoon, J. W.; Seo, Y. K.; Hwang, Y. K.; Chang, J. S.; Leclerc, H.; Wuttke, S.; Bazin, P.; Vimont, A.; Daturi, M.; Bloch, E.; et al. Controlled Reducibility of a Metal-Organic Framework with Coordinatively Unsaturated Sites for Preferential Gas Sorption. Angew. Chemie - Int. Ed. 2010, 49 (34), 5949-5952.

(24) Giménez-Marqués, M.; Hidalgo, T.; Serre, C.; Horcajada, P. Nanostructured Metal-Organic Frameworks and Their Bio-Related Applications. Coord. Chem. Rev. 2016, 307, 342-360.

(25) Knowles, J. R.; Roller, S. Antimicrobial Action of Carvacrol at Different Stages of Dual-Species Bio Lm Development By. Society 2005, 71 (2), 797-803. 
(26) Liolios, C. C.; Gortzi, O.; Lalas, S.; Tsaknis, J.; Chinou, I. Liposomal Incorporation of Carvacrol and Thymol Isolated from the Essential Oil of Origanum Dictamnus L. and in Vitro Antimicrobial Activity. Food Chem. 2009, $112(1), 77-83$.

(27) Ramos, M.; Jiménez, A.; Peltzer, M.; Garrigós, M. C. Characterization and Antimicrobial Activity Studies of Polypropylene Films with Carvacrol and Thymol for Active Packaging. J. Food Eng. 2012, 109 (3), 513-519.

(28) Kamimura, J. A.; Santos, E. H.; Hill, L. E.; Gomes, C. L. Antimicrobial and Antioxidant Activities of Carvacrol Microencapsulated in Hydroxypropyl-Beta-Cyclodextrin. LWT - Food Sci. Technol. 2014, 57 (2), 701-709.

(29) He, J.; Huang, S.; Sun, X.; Han, L.; Chang, C.; Zhang, W.; Zhong, Q. Carvacrol Loaded Solid Lipid Nanoparticles of Propylene Glycol Monopalmitate and Glyceryl Monostearate: Preparation, Characterization, and Synergistic Antimicrobial Activity. Nanomaterials 2019, 9 (8), 1-14.

(30) Hesse, J.; Rubartsch, A. Model Independent Evaluation of Overlapped Mossbauer Spectra. J. Phys. E. 1974, 7 (7), 526-532.

(31) Higueras, L.; López-Carballo, G.; Gavara, R.; Hernández-Muñoz, P. Effect of Hydroxypropyl- $\beta$ Cyclodextrin and Coadjuvants on the Sorption Capacity of Hydrophilic Polymer Films for Monoterpene Alcohols. Carbohydr. Polym. 2016, 151, 1193-1202.

(32) M. J. Frisch, G. W. Trucks, H. B. Schlegel, G. E. Scuseria, M. A. Robb, J. R. Cheeseman, G. Scalmani, V. Barone, G. A. Petersson, H. Nakatsuji, X. Li, M. Caricato, A. V. Marenich, J. Bloino, B. G. Janesko, R. Gomperts, B. Mennucci, H. P. Hratchian, J. V., and D. J. F. Gaussian 16, Revision A.03. Gaussian, Inc.: Wallingford CT 2016.

(33) Becke, A. D. Density-Functional Thermochemistry. III. The Role of Exact Exchange. J. Chem. Phys. 1993, 98 (7), 5648-5652.

(34) Rassolov, V. A.; Ratner, M. A.; Pople, J. A.; Redfern, P. C.; Curtiss, L. A. 6-31G* Basis Set for Third-Row Atoms. J. Comput. Chem. 2001, 22 (9), 976-984.

(35) Grimme, S.; Antony, J.; Ehrlich, S.; Krieg, H. A Consistent and Accurate Ab Initio Parametrization of Density Functional Dispersion Correction (DFT-D) for the 94 Elements H-Pu. J. Chem. Phys. 2010, 132 (15), 154104.

(36) Grimme, S.; Ehrlich, S.; Goerigk, L. Effect of the Damping Function in Dispersion Corrected Density Functional Theory. J. Comput. Chem. 2011, 32, 1456-1465. 
(37) Contreras-García, J.; Johnson, E. R.; Keinan, S.; Chaudret, R.; Piquemal, J. P.; Beratan, D. N.; Yang, W. NCIPLOT: A Program for Plotting Noncovalent Interaction Regions. J. Chem. Theory Comput. 2011, 7 (3), 625-632.

(38) Humphrey, W.; Dalke, A.; Schulten, K. VMD: Visual Molecular Dynamics. J. Mol. Graph. 1996, 14 (October 1995), 33-38.

(39) Chemcraft - graphical software for visualization of quantum chemistry computations https://www.chemcraftprog.com.

(40) Monteagudo-Olivan, R.; Cocero, M. J.; Coronas, J.; Rodríguez-Rojo, S. Supercritical CO 2 Encapsulation of Bioactive Molecules in Carboxylate Based MOFs. J. CO2 Util. 2019, 30 (December 2018), 38-47.

(41) Maryam, K.; Shakeri, S.; Kiani, K. Preparation and in Vitro Investigation of Antigastric Cancer Activities of Carvacrol-Loaded Human Serum Albumin Nanoparticles. IET Nanobiotechnology 2015, 9 (5), 294-299.

(42) Leclerc, H.; Vimont, A.; Lavalley, J. C.; Daturi, M.; Wiersum, A. D.; Llwellyn, P. L.; Horcajada, P.; Férey, G.; Serre, C. Infrared Study of the Influence of Reducible Iron(Iii) Metal Sites on the Adsorption of CO, CO2, Propane, Propene and Propyne in the Mesoporous Metal-Organic Framework MIL-100. Phys. Chem. Chem. Phys. 2011, 13 (24), 11748-11756.

(43) Bellido, E.; Hidalgo, T.; Lozano, M. V.; Guillevic, M.; Simón-Vázquez, R.; Santander-Ortega, M. J.; González-Fernández, Á.; Serre, C.; Alonso, M. J.; Horcajada, P. Heparin-Engineered Mesoporous Iron Metal-Organic Framework Nanoparticles: Toward Stealth Drug Nanocarriers. Adv. Healthc. Mater. 2015, $4(8), 1246-1257$.

(44) Hidalgo, T.; Alonso-Nocelo, M.; Bouzo, B. L.; Reimondez-Troitiño, S.; Abuin-Redondo, C.; De La Fuente, M.; Horcajada, P. Biocompatible Iron(Iii) Carboxylate Metal-Organic Frameworks as Promising RNA Nanocarriers. Nanoscale 2020, 12 (8), 4839-4845.

(45) Yoon, J. W.; Seo, Y. K.; Hwang, Y. K.; Chang, J. S.; Leclerc, H.; Wuttke, S.; Bazin, P.; Vimont, A.; Daturi, M.; Bloch, E.; et al. Controlled Reducibility of a Metal-Organic Framework with Coordinatively Unsaturated Sites for Preferential Gas Sorption. Angew. Chemie - Int. Ed. 2010, 49 (34), 5949-5952.

(46) Greenwood, N.N., Gibb, T. C. Mössbauer Spectroscopy; Hall, C. and, Ed.; Ltd. Publishers: London, 1971.

(47) Gavrilenko, K. S.; Vértes, A.; Vanko, G.; Kiss, L. F.; Addison, A. W.; Weyhermüller, T.; Pavlishchuk, V. V. Synthesis, Magnetochemistry, and Spectroscopy of Heterometallic Trinuclear Basic 
Trifluoroacetates [Fe2M(M3-O)(CF3COO)6 (H2O)3] $\mathrm{H} 2 \mathrm{O}(\mathrm{M}=\mathrm{Mn}, \mathrm{Co}, \mathrm{Ni})$. Eur. J. Inorg. Chem. 2002, 6 (12), 3347-3355.

(48) Ramos Silva, M.; Coutinho, J. T.; Pereira, L. C. J.; Martín-Ramos, P.; Waerenborgh, J. C. Synthesis, Structure and Physical Properties of a Low Dimensional Compound. Spectrochim. Acta - Part A Mol. Biomol. Spectrosc. 2017, 172, 9-13. 